\title{
Transitional cell carcinoma of ureter presenting acute ureteric colic and ruptured caliceal fornix with extravasation of urine
}

\author{
Phornthep Ruchdaphornkul, Kamol Panumatrassamee \\ Division of Urology, Department of Surgery, Faculty of Medicine, Chulalongkorn University, Bangkok \\ 10330, Thailand
}

\begin{abstract}
Backgroud: Primary ureteral tumors are not common. The most common presentations of upper urinary tract urothelial carcinoma are gross or microscopic hematuria.

Objective: Report a rare case of acute ureteric colic with perirenal urinoma, secondary to primary ureteric carcinoma. Methods: Case report and review of literature.

Result: Perirenal extravasation and ureteric obstruction by tumor was diagnosed by contrast-enhanced computed tomography. The patient was successfully treated with laparoscopic radical nephroureterectomy after the obstruction was relieved by ureteric stent. Adjuvant radiation was done at the renal fossa for local control. There was no evidence of disease recurrence at twelve month after surgery.

Conclusion: The renal caliceal fornix rupture is usually associated with acute ureteric obstruction by stone. Rarely, it occurs by primary ureteric tumor.
\end{abstract}

Keywords: Acute ureteric colic, perirenal urinoma, ruptured caliceal fornix, ureteric carcinoma

Spontaneous rupture of the renal calyceal fornix with urine extravasation is usually associated with ureteric obstruction by urinary calculi [1]. Patients often have a sudden onset of abdominal pain radiating to the pubic symphysis. Rarely, this presentation is found with malignant tumors $[2,3]$. We report a case of spontaneous renal caliceal fornix rupture due to urothelial carcinoma of the ureter. The rarity of this condition poses challenges in its diagnosis and management.

\section{Case report}

A 77 years-old diabetic non-smoking woman was admitted by the emergency room because she had an acute onset of right-side abdominal pain. The symptom started during non-strenuous exercise without radiating pain. She had nausea but no vomiting and

Correspondence to: Ruchdaphornkul P, Division of Urology, Department of Surgery, Faculty of Medicine, Chulalongkorn University, Bangkok 10310, Thailand. E-mail: Phornthep@ windowslive.com no fever or chills. There was no history of hematuria or stone disease. Her symptoms had persisted for three days before she decided to seek medical care. On admission, her body temperature was $37.9^{\circ} \mathrm{C}$ and her blood pressure was $142 / 62 \mathrm{mmHg}$. Abdominal examination revealed tenderness and guarding at the right upper abdominal area without palpable mass. The right costovertebral angle was tender on percussion. Her complete blood count showed leucocytosis (white blood cell 22,710 cells $/ \mathrm{mm}^{3}$ with neutrophil predominate). The urinalysis was unremarkable. Her BUN/Cr was 16/1.0, SGOT was 25, SGPT was 47, ALP was 95 , TB was 0.95 , and DB was 0.5 .

The provisional diagnosis at the emergency room was acute cholecystitis. Computer tomography revealed moderate right hydronephrosis and hydroureter down to the mid-ureter. There was contrast media extravasated into the right perinephric space. Contrast media did not pass into the right distal ureter due to obstruction and perirenal leakage. Liver, gall bladder, and other intraperitoneal organ were normal (Fig. 1, 2) 


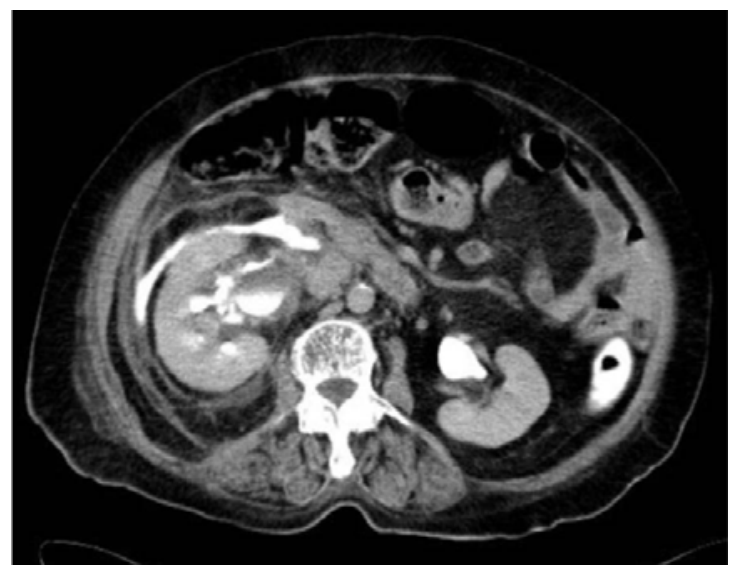

Fig.1 Delayed phase of contrast-enhanced CT scanning that revealed extravasation of urine surrounding the right perinephric space with the right moderate hydronephrosis.

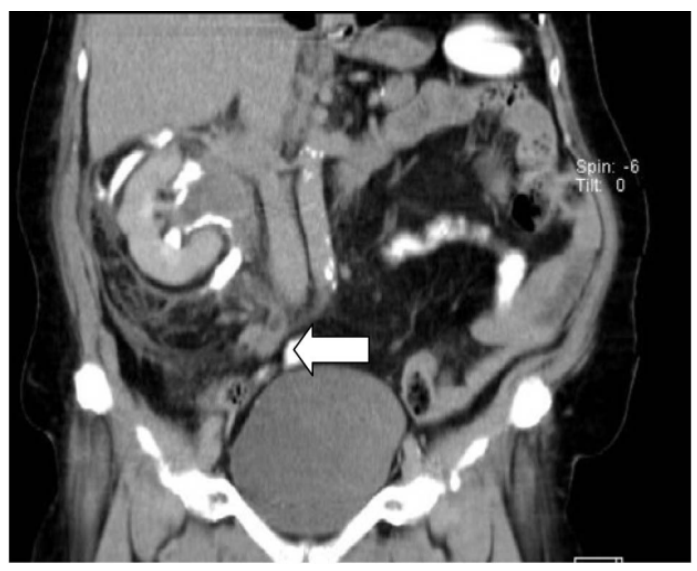

Fig. 2 Coronal view of CT scanning to show the right hydroureter down to the ureteric mass (indicated by an arrow).

Initially, the cause of obstruction was not identified and the patient was referred to the urology department. A retrograde pyelogram was performed and revealed that the right ureter was completely obstructed at mid-ureteric level. Retrograde placement of a double $\mathrm{J}$ stent was attempted but unsuccessful because of obstruction. Ureterorenoscope was preformed to identify the cause of obstruction and showed a large mass in right mid ureter- $15 \mathrm{cms}$ from right ureteric orifice. Cold cup biopsy was done, and a right ureteric stent (double J stent, No.4.7 Fr) was inserted. After the operation, her symptom improved dramatically. Histopathology revealed a high-grade urothelial carcinoma. Laparoscopic radical nephroureterectomy with bladder cuff excision was performed three weeks later.

Gross pathology revealed a two-centimeter ureteric mass with nearly complete obstruction of the ureter without renal pelvic mass (Fig. 3). The final pathological report confirmed that she had high-grade urothelial carcinoma with muscularis propria and angiolymphatic invasion without nodal metastasis (T2N0M0). The patient received adjuvant radiation at the right renal fossa due to high risk for local recurrence from the urine extravasation.

\section{Discusion}

Upper urinary tract urothelial tumors involving the renal pelvis or ureter are relatively uncommon. They account for about $5 \%$ of all urothelial tumors. Men are two times more likely to develop upper tract tumors than women [4]. Several previous reports stated that ureteric tumors have a poorer overall prognosis. The five-year disease-specific survival and recurrence-free survival is poor because the thinning wall of the ureter eases tumor spread compared with tumors in renal 


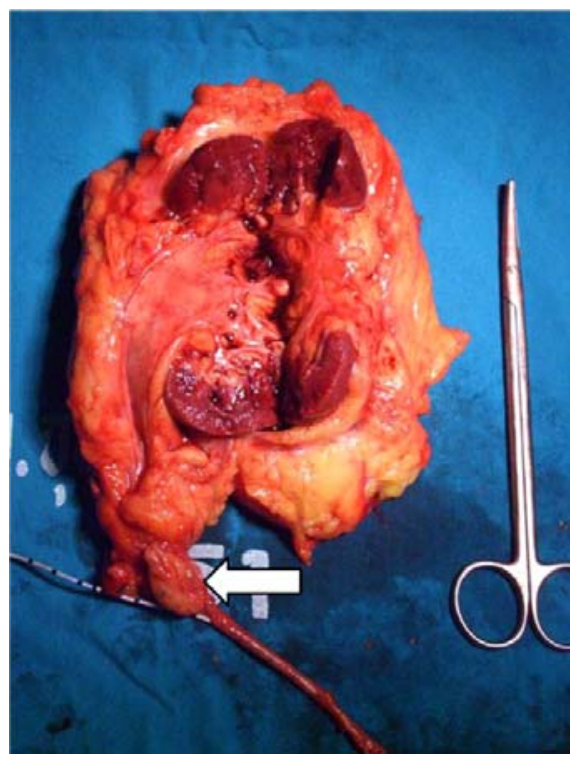

Fig. 3 Gross surgical pathology. Tumors are indicated by an arrow.

pelvis [5]. The overall prognosis of upper tract tumors seems to be related to tumor stage rather than tumor grade. Lymphovascular invasion was also the independent prognosis factor for disease specific survival. The most common presenting symptoms of upper tract urothelial tumor are gross or microscopic hematuria. In rare cases, the patient may present with acute flank pain that mimics renal colic, which can delay early diagnosis and proper management. Computed tomographic urography is increasingly used to distinguishing between upper tract urothelial carcinoma and stone. Urothelial carcinoma has an average density of 46 Hounsfield units (HU) (range: 10 to $70 \mathrm{HU}$ ) [6], but stone is usually higher.

Despite the fact that renal caliceal fornix rupture is usually associated with acute ureteric obstruction by stone, it could also be obstructed by primary ureteric tumor. Reviewing the literature in Pubmed, we found 11 cases of ureteric cancer presenting as spontaneous rupture of the calyceal fornix reported from Japan between 1995 and 2009 [7-9]. Most of these cases were diagnosed by CT scan and managed surgically.

In our case, the patient presented acute onset of abdominal pain that had signs of localized peritonitis with normal urinalysis. Her presenting symptoms mimic other surgical conditions, such as acute cholecystitis or atypical appendicitis. Contrast enhanced CT scan revealed the ruptured renal caliceal fornix with extravasation caused by ureteric tumor obstruction. Urinary drainage was performed to relieve obstruction and the patient symptoms. Applying on internal stent appeared to be the first choice for preventing further tumor spillage from urine leakage.

Although radical nephroureterectomy is the gold standard for control of localized transitional cell carcinoma of the upper urinary tract, the optimal treatment of high-grade cancer with urinary extravasation into the perinephric space was not discussed in the current literature. Although there is little guidance due to the small number of cases reported, we decided to carry out adjuvant radiation. The urinary extravasation was confined within Gerota's fascia in our patient, and we were concerned about local recurrence of disease. She had an uncomplicated course, appearing to be well at twelve month follow-up.

The authors have no coflict of interest to report.

\section{References}

1. Paajanen H, Kettunen J, Tainio H, Jauhiainen K. Spontaneous peripelvic extravasation of urine as a cause of acute abdomen. Scand J Urol Nephrol. 1993; 27:333-6.

2. Koga S, Arakaki Y, Matsuoka M, Ohyama C. Spontaneous peripelvic extravasation of urine. Int Urol Nephrol. 1992; 24:465-9.

3. Spurlock JW, Burke TW, Dunn NP, Heller PB, Collins HS, Park RC. Calyceal rupture with perirenal urinoma in a patient with cervical carcinoma. Obstet Gynecol. 1987; 70:511-3. 
4. Greenlee RT, Murray T, Bolden S, Wingo PA. Cancer statistics, 2000. CA Cancer J Clin. 2000; 50:7-33.

5. Park S, Hong B, Kim CS, Ahn H. The impact of tumor localization on prognosis of transitional cell carcinoma of the upper urinary tract. J Urol. 2004; 171:621.

6. Lantz EJ, Hattery RR. Diagnostic imaging of urothelial cancer. Urol Clin North Am. 1984; 11:576.

7. Miyajima A, Ikeuchi K. Spontaneous rupture of ureter caused by ureteral cancer (in Japanese). Nippon Hinyokika Gakkai Zasshi. 1995; 86:1789-92.
8. Noda Y, Tujikawa K, Takada S, Sugao H, Itou Y, Osafune M. Spontaneous renal rupture resulting from ureteral tumor left untreated for 6 years: a case report (in Japanese). Hinyokika Kiyo. 2001; 47:265-8.

9. Inahara M, Kojima S, Takei K, Naito H, Kito H, Yamazaki $\mathrm{K}$, et al. Two cases of spontaneous rupture of upper urinary tract caused by the primary ureteral or renal pelvic tumor: a case report (in Japanese). Hinyokika Kiyo. 2009; 55:31-4. 